\title{
Electrical stimulation for difficult wounds: only an alternative procedure?
}

\author{
Marco Fraccalvieri, Marco Salomone, Enrico M Zingarelli, Filippo Rivarossa \& Stefano Bruschi \\ Department of Plastic, Reconstuctive and Aesthetic Surgery, University of Turin, Torino, Italy
}

\author{
Key words \\ BST; Electrical stimulation; Pain; Stochastic \\ resonance; Wound healing \\ Correspondence to \\ M Fraccalvieri \\ Department of Plastic, Reconstuctive and \\ Aesthetic Surgery \\ University of Turin \\ Via Cherasco 23 \\ Turin 10100 \\ Italy \\ E-mail: marco@fraccalvieri.it
}

doi: 10.1111/iwj.12194
Fraccalvieri M, Salomone M, Zingarelli EM, Rivarossa F, Bruschi S. Electrical stimulation for difficult wounds: only an alternative procedure? Int Wound J 2015; $12: 669-673$

\section{Abstract}

In the wound healing research, the exact mechanism of action of different modalities of electrical stimulation (ES) remains controversial and unresolved. In this study we discuss a particular ES, with a different type of waveform, corresponding to the principle of stochastic resonance. Between July 2008 and May 2010, 32 patients were enrolled and ES was applied to wounds using the bioelectrical signal therapy (BST) device (LifeWave, Petach Tiqwa, Israel). The outcome evaluated in group $1(n=21)$ was wound healing, while group $2(n=11)$ was evaluated for wound-related pain [Visual Number Scale (VNS) pain scale] during treatment. In group 1, 87\% of the wounds closed in an average time of 97 days (range 10-150 days); three patients were lost to follow-up. In group 2, $45 \%$ of the patients experienced a complete pain disappearance after 7 days of treatment; $36 \%$ reported a reduction in VNS from 9.3 to 3.2 in 7 days; $19 \%$ stopped morphine-like painkillers after 2 weeks. The clinical application of the stochastic resonance enables the usage of easy-to-use, non-invasive, painless and pain-relief treatment. Our experience with ES has demonstrated the BST device to be a very good alternative in cases of small size defects, compared with other therapies such as surgery, dressing and negative pressure devices.

\section{Introduction}

The first empirical electrical stimulation (ES) dates back to the 17 th century, where gold leafs were used in the treatment of cutaneous ulcer (1). The first researches on human endogenous bioelectric circuits were demonstrated only in 1843, when DuBois-Reymond described the electrical currents within a human skin wound (2). Then, a map of human 'skin battery' voltages was presented $(3,4)$.

Intact human skin has an endogenous potential ranging from 10 to $60 \mathrm{mV}$, maintained by $\mathrm{Na} / \mathrm{K}$ ATPase; in chronic wounds there is a loss of this physiological current $(5,6)$. These findings have led researchers and clinicians to examine the use of various treatments, in particular different forms of electrostimulation in chronic wound healing, in order to reestablish this endogenous potential.

Consequently, the use of ES as an additional treatment (and prevention $(7,8)$ ) for chronic wounds of various aetiology has been explored (9-11) including burns, diabetic foot ulcers, venous, mixed and arterial leg ulcers and pressure

\section{Key Messages}

- intact human skin has an endogenous potential ranging from 10 to $60 \mathrm{mV}$, maintained by $\mathrm{Na} / \mathrm{K}$ ATPase and in chronic wounds there is a loss of this physiological current

- the success of electrical stimulatio (ES) is best observed when treating wounds unresponsive to conventional therapy

- this case series focus on the effects of a particular and different type of ES, based on the recent application of a different type of waveform, corresponding to the principle of stochastic resonance

- in stochastic resonance, sensory neurons can significantly enhance their ability to detect weak stimuli by addition of an appropriate amount of noise

- electrostimulation has demonstrated to reduce pain related to wounds 
ulcers. The success of ES is best observed when treating wounds unresponsive to conventional therapy (8). Nowadays, international pressure ulcer guidelines [European Pressure Ulcer Advisory Panel (EPUAP) and National Pressure Ulcer Advisory Panel (NPUAP)] (12,13) rate ES as the only treatment for pressure ulcers with the highest strength of evidence.

The exact mechanism of action of different modalities of ES remains controversial and unresolved.

Different types of electrical current are mainly used: direct current (DC), low-frequency pulsed current, highvoltage pulsed current, pulsed electromagnetic fields (2) and alternating current.

In this study we discuss a particular type of ES, based on the recent application of a different type of waveform, corresponding to the principle of stochastic resonance introduced in this type of treatment.

Moreover, we suggest using this type of waveform to manage pain associated with chronic wounds, as this device stimulates the peripheral neural system and shows an improvement in sensitive perception.

\section{Method}

\section{Setting and design}

The study took place in the Plastic Surgery Department of the University of Torino, Chronic Wounds Outpatient Clinic. The inclusion criteria were patients with chronic wounds not responding to traditional dressings, surgery or negative pressure therapies (NWPT) for at least 4 weeks, with a wound size up to $20 \mathrm{~cm}^{2}$. The study was conducted in a 22-month period between July 2008 and May 2010.

The patients were divided into two groups to evaluate two different outcomes: wound healing and pain. The aetiology of each wound was considered.

Exclusion criteria in both groups were:

- Pregnancy or breastfeeding

- Patients with arrhythmia, active pacemaker or defibrillator, kidney failure, liver failure, severe anaemia or sepsis

- Wounds at chest level or above

- Patients with neoplastic lesions

- Patients with silver dressings

The first group is composed of patients with wounds that were not responding to conventional therapies for at least 3 months. This group was evaluated for the wound healing. In order to monitor the progression of the wound, an integrating system composed of digital camera (for wound dimension), a laser and a software (for wound depth) was used. Data on the skin surrounding the wound were also acquired.

The second group is composed of patients with severe pain [with Visual Number Scale (VNS) > 8] greatly impairing their quality of life or requiring a heavy use of painkillers. This group was evaluated for the effect of electric therapy on pain control; in order to evaluate the pain progression, VNS scale was used and data were acquired before, during and after the treatment.

\section{Device}

This study was focused on the effects of the bioelectrical signal therapy device (BST, LifeWave, Petach Tiqwa, Israel) on chronic wounds. This is a single channel electrical stimulator, composed of a main computer with the circuits and the user interface, and two electrodes to apply on the skin surrounding the wound. There is no need to place the electrodes directly on the wound because this device works with alternate current rather than DC: the optimum placement of the electrodes is 3 or $5 \mathrm{~cm}$ from the border of the wound, depending on the condition of the skin surrounding the wound. It is the only approved electrical therapy on chronic wounds in the European Union (EU), Canada, Brazil and Israel.

\section{ES waveform}

The stimulation mode is a low-frequency $(2 \mathrm{~Hz})$ periodic pulse sequence composed of two integrated waveforms; a rectangular pulse train and a stochastic (random) signal. Following the digital synthesis and integration of the two signals (and their conversion to analogue signals), the integrated waveforms are filtered by a low pass filter at $2500 \mathrm{~Hz}$.

The stochastic waveform component originates from a digital precursor that is a white signal (i.e. the same intensity range exists along the frequency spectrum). The output of the stochastic signal has a maximum amplitude of $7.5 \pm 0.5 \mathrm{~V}$ (on $2 \mathrm{k} \Omega$ ), duration of 0.246 seconds with $80 \%$ of energy in the bandwidth of $0-1300 \mathrm{~Hz}$, along this bandwidth the signal exhibits a stochastic white waveform. The output of the rectangular pulse train is a periodic biphasic pulse which has maximum amplitude of $12 \pm 1 \mathrm{~V}$ (on $2 \mathrm{k} \Omega$ ) and pulse duration of 4 milliseconds.

\section{Treatment}

Participants were treated with the BST device thrice daily, for 30 minutes each. The patients were taught to use the device, so that the treatment could be provided at home without external help. No difficulties in the use of the device were reported. All patients were seen once a week in the Chronic Wounds Outpatient Clinic to check the progression.

The standard wound therapy continued as required, including standard wound care with medications. The management of the dressings was provided as recommended by the manufacturer: hydrocolloids, hydrogels foams, alginate and other dressings were used and changed when necessary following the standard procedure. Advanced therapies such as NPT and surgical procedures were provided when required.

Patients with dressing having metallic traces (such as silver dressings) were excluded because of a possible interaction of the metal particles of the dressing with the electrical fields generated by the device.

The study protocol conformed to the ethical guidelines of the 1975 Declaration of Helsinki 


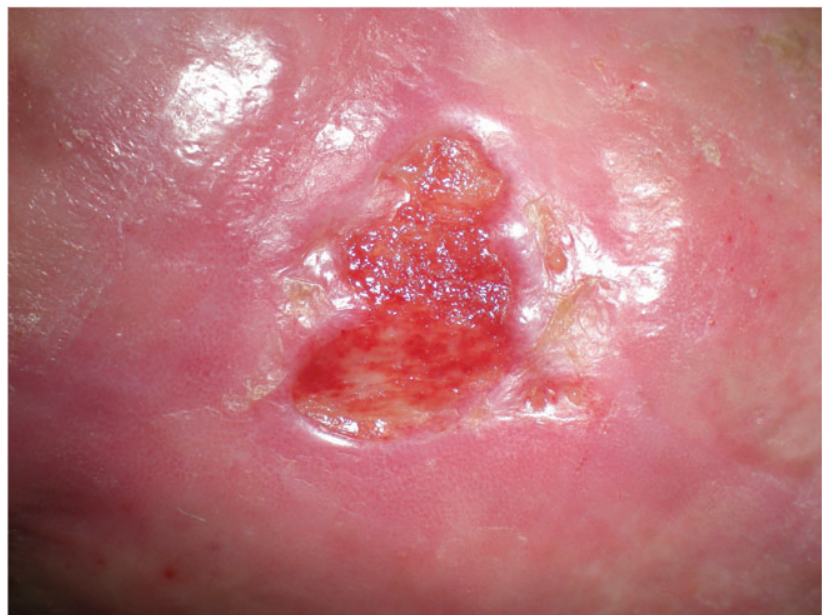

Figure 1 Pre-treatment appearance of the lesion; 61-year-old male, with mixed circulatory insufficiency. Previous treatment included skin grafts with partial respond. Wound dimension $3 \mathrm{~cm} \times 2 \mathrm{~cm}$

\section{Results}

\section{Wound healing group}

In the first group, 21 patients were included (aged 38-88, average 69$) ; 15$ were males $(71 \%)$ and 6 were females $(29 \%)$. The aetiology of the wounds in this first group was: diabetes $24 \%$, arterial wounds $24 \%$, pressure $19 \%$, trauma $14 \%$, venous wounds $10 \%$ and scleroderma $10 \%$. The average size of the wounds was $4.92 \mathrm{~cm}^{2}$, ranging from 0.8 to $12 \mathrm{~cm}^{2}$. Out of 21 cases $15(71 \%)$ went into a change from chronic wounds to acute wounds in an average time of 34 days, with a range of treatment period from 3 to 70 days.

Three patients (14\%) were lost to follow-up owing to complications or low compliance to the treatment. Two patients $(10 \%)$ did not respond to the treatment. At the end of the follow-up period, $87 \%$ of the wounds were completely closed in an average time of 97 days (range 10-150 days), while $13 \%$ were still in reepithelialisation.

In Figures 1-6 the history of the wound healing with BST device is described.

\section{Pain control group}

In the second group, 11 patients were included (aged 38-84, average 67), 5 males and 6 females. The aetiology of the wounds in this second group was: diabetes 33\%, arterial wounds $25 \%$, venous wounds $17 \%$, scleroderma $17 \%$ and trauma $8 \%$. The average VNS before treatment was 8.7 (range 8-10).

After just 7 days of treatment, five patients (45\%) reported a complete pain disappearance, with pain level decreasing from an average VNS of 8.5 to VNS 1 .

Of the latter six patients, four (36\%) reported a similar reduction with a decrease of the average VNS from 9.3 to 3.2 in the same 7-day period. The latter two patients (19\%) had very severe pain that required daily treatment with morphinelike painkillers. Both patients reported a strong pain reduction from an average VNS of 9.5 to VNS 2.5 after just 1 week of

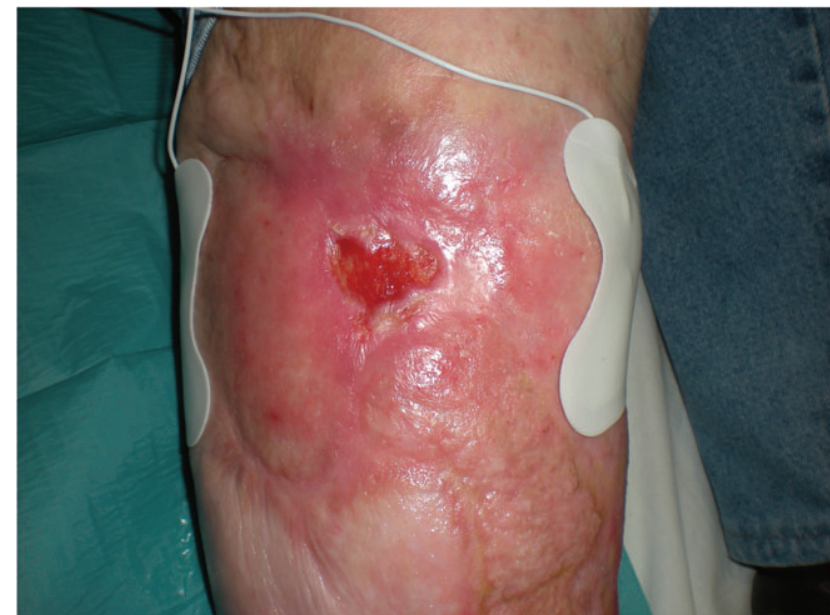

Figure 2 Same patient in Figure 1, during treatment. Bioelectrical signal therapy (BST) device applied close to the wound. During treatment, no other type of advanced dressing was used.

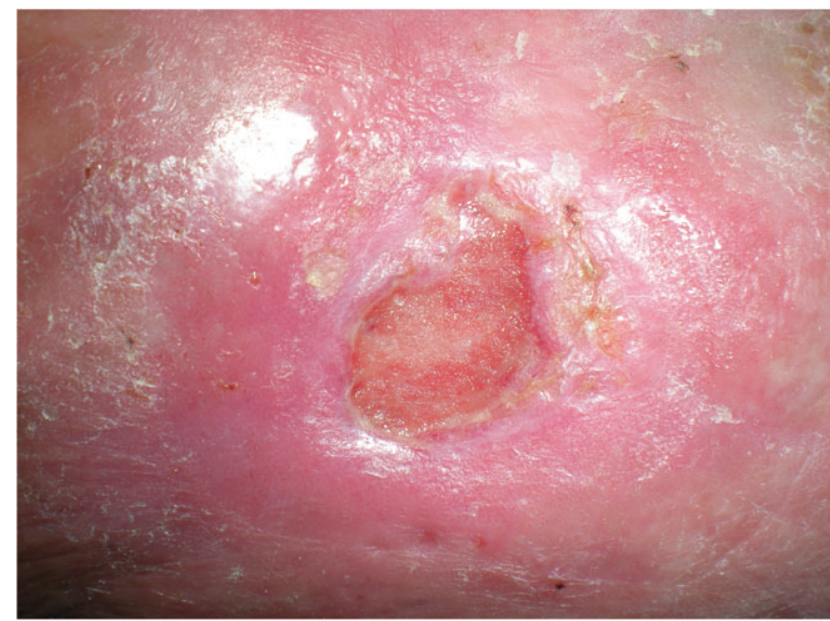

Figure 3 Wound dimension $2.5 \mathrm{~cm} \times 1.5 \mathrm{~cm}$ after 30 days of treatment.

treatment, that allowed them to reduce the dose of painkillers first, and then to stop them definitely after 2 weeks.

\section{Discussion}

The exact mechanism of action of different modalities of ES remains controversial and unresolved.

Different types of current are mainly used: DC, lowfrequency pulsed current, high-voltage pulsed current, pulsed electromagnetic fields (2) and alternating current.

Some DC or pulsed monophasic waveforms work primarily by activating galvanotaxis (14). Galvanotaxis is the property of keratinocytes clusters to migrate along the potential gradient towards the wound, attracted by positively or negatively charged cells towards an electric field of opposite polarity. (15) This phenomenon enhances migration of leucocytes, fibroblasts and allows reepithelisation (11).

Other alternating currents activate cutaneous sensory nerves (14), which may play an important role in tissue repair, by 


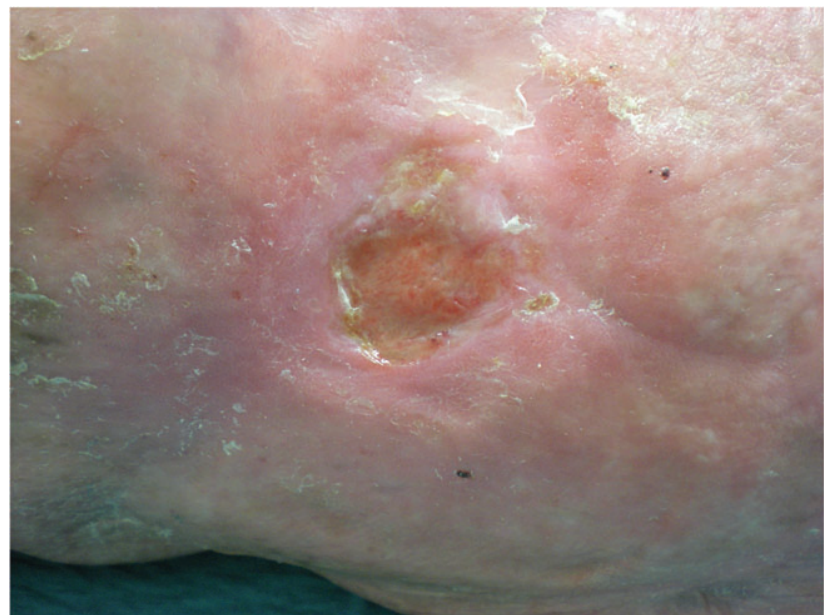

Figure 4 Wound dimension $1.5 \mathrm{~cm} \times 1.5 \mathrm{~cm}$ after 50 days of treatment. Treatment with bioelectrical signal therapy (BST) is stopped.

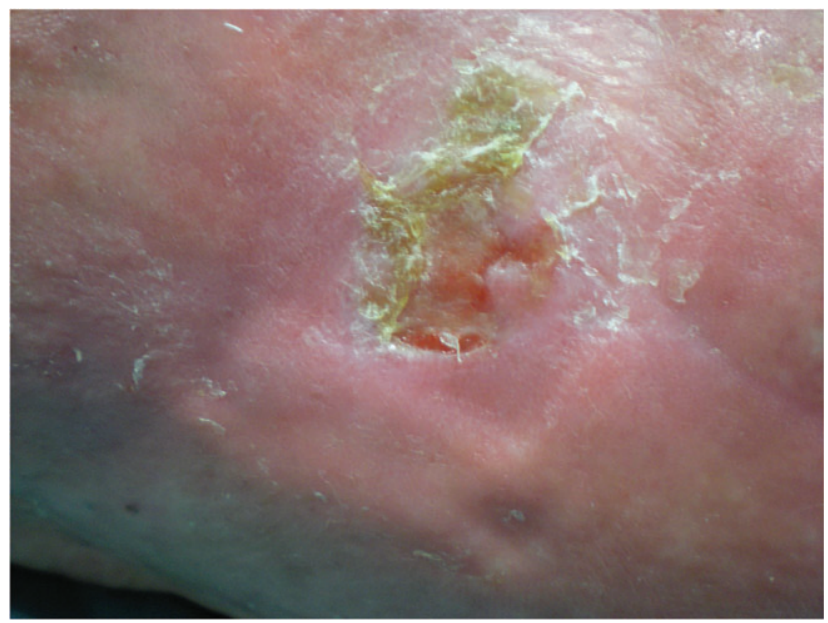

Figure 5 Eighty days follow-up after bioelectrical signal therapy (BST) was started; 30 days after BST treatment was suspended hydrocolloids were applied.

increasing blood flow and the sensitivity of the injured tissue around wounds.

Other attributes (11) of electrostimulation were described: (i) DNA and protein synthesis (upregulation of insulin and TGF- $\beta$ receptors on fibroblasts, (ii) antibacterial effects by imposing a bacteriostatic or bactericidal effect on microbes that commonly colonise or infect wounds (presence of silver cations deposited in vivo, $\mathrm{pH}$ changes, electrophoretic recruitment of antimicrobial factors, local heat generation) and (iii) angiogenesis, tissue oxygenation and activation of the complement cascade.

This case series focus on the effects of a particular and different type of ES, based on the recent application of a different type of waveform, corresponding to the principle of stochastic resonance.

The ES of the BST derives from the combination of a rectangular pulse train and a stochastic (random) signal to create a 'noise'. This noise is an integral part of the normal

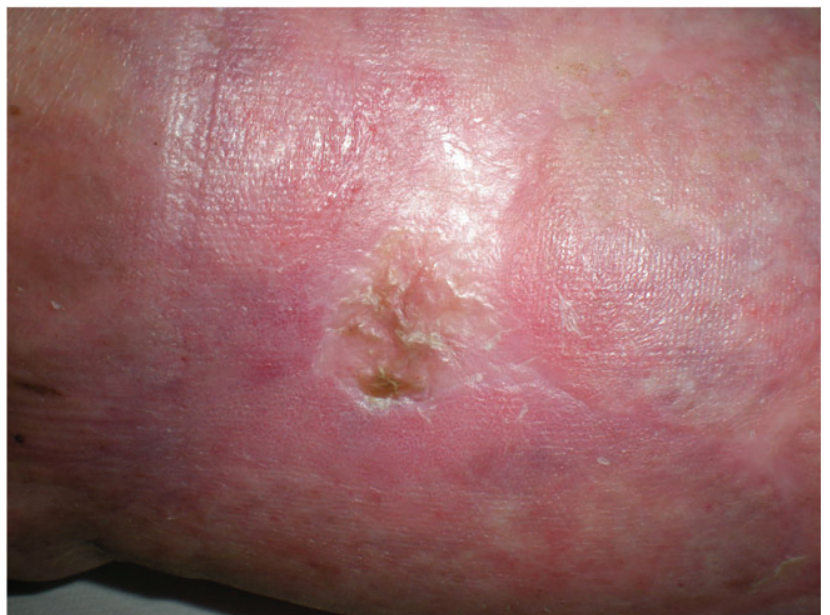

Figure 6 One hundred and twenty days after bioelectrical signal therapy (BST) positioning. As shown, complete reepitelisation can be achieved even 2 months after suspension of BST treatment, due to the reactivation of physiological ion current within the wound.

sensory process. When this phenomenon of noise improves sensory performance, it is termed stochastic resonance (16), where sensory neurons can significantly enhance their ability to detect weak stimuli by addition of an appropriate amount of noise (17).

Collins in 1965 described the stochastic resonance, but its current application with BST device is recently introduced $(18,19)$.

There is only a multicentre controlled randomised study on grade III pressure ulcers that describe the safety and efficacy of BST: with a treatment period of 57 days, $50 \%$ of patients in the BST group who had ulcers above the knee achieved complete closure compared with $9 \%$ in the placebo group ( $P=0.035)$ (19).

A more recent case series reviewed the efficacy of BST on various aetiology of hard to heal wounds with the worst case scenario (14); the greatest success of ES is best observed when treating wounds unresponsive to conventional therapy (8). In fact in this study we used ES with BST device only when surgery, traditional and advanced wound care failed to reach the goal.

In our series, ES treatment lasted for long periods until complete wound healing. The treatment should be stopped as the wound changes from chronic to acute phase; subsequently, wound care should be continued with conventional or other advanced therapies.

The human body creates a bioelectrical stochastic noise that is compromised in chronic wounds. The BST goal is to reestablish this physiological phenomenon, reconnecting the wound to the peripheral nervous system (PNS). Injuries to the PNS or CNS (central nervous system) may partially reduce the efficacy of this type of treatment, as observed by Ricci (13).

The importance and the effects of the restabilisation of the stochastic noise on the nervous system can be observed in the efficacy of BST to reduce pain or, for example, in enhanced sensation in patients with diabetic neuropathy (20-22). There 
is a growing awareness on the importance of treating patient's pain as it is frequently experienced in patients with leg ulcers, often chronic, and subjected frequently to wound relapse (23). Electrostimulation has demonstrated to reduce pain related to wounds (15).

Various factors may contribute to the pain relief, for example, 'local' phenomena such as increased tissue oxygenation, reestablished $\mathrm{pH}$, oedema and reduced of inflammatory agents.

On the other hand, a potential 'distant' factor that contributes to the pain relief may be hypothesised in the stimulation of the nervous system: as described in headache disorders the stimulation of sensory $\beta$-fibres close the 'pain gate control' by inhibition of the stimuli coming to the CNS from nociceptive $C$ fibres. In literature, this mechanism of action is not yet described for the ES applied by BST, and it could be a topic of future researches.

\section{Conclusion}

The clinical application of the stochastic resonance enables the use of an easy-to-use, non-invasive, painless and pain relief treatment.

In our study, ES with BST device has been demonstrated to be better than a good alternative in cases of small size defects, compared with other therapies such as surgery, dressings and NWPT

\section{References}

1. Karnof N. Gold leaf in treatment of cutaneous ulcers. $J$ Invest Dermatol 1964;43:441-2.

2. Hess C, Howard M, Attinger C. A review of mechanical adjuncts in wound healing: hydrotherapy, ultrasound, negative pressure therapy, hyperbaric oxygen, and electrostimulation. Ann Plast Surg 2003;51:210-8.

3. Foulds I, Barker A. Human skin battery potentials and their possible role in wound healing. Br J Dermatol 1983;109:515-22.

4. Barker A, Jaffe L, Vanable JJ. The glabrous epidermis of cavies contains a powerful battery. Am J Physiol 1982;242:R358-66.

5. Baker L, Chambers R, DeMuth S, Villar F. Effects of electrical stimulation on wound healing in patients with diabetic ulcers. Diabetes Care 1997;20:405-12.

6. Nishimura $\mathrm{K}$, Isseroff $\mathrm{R}$, Nuccitelli $\mathrm{R}$. Human keratinocytes migrate to the negative pole in direct current electric fields comparable to those measured in mammalian wounds. J Cell Sci 1996;109:199-207.
7. Kim J, Ho C, Wang X, Bogie K. The use of sensory electrical stimulation for pressure ulcer prevention. Physiother Theory Pract 2010;26:528-36.

8. Bogie K, Reger S, Levine S, Sahgal V. Electrical stimulation for pressure sore prevention and wound healing. Assist Technol 2000;12:50-66.

9. Gardner S, Frantz R, Schmidt F. Effect of electrical stimulation on chronic wound healing: a meta-analysis. Wound Repair Regen 1999; 7:495-503.

10. Sheffet A, Cytryn A, Louria D. Applying electric and electromagnetic energy as adjuvant treatment for pressure ulcers: a critical review. Ostomy Wound Manage 2000;46:28-33, 36-40, 42-4.

11. Kloth L. Electrical stimulation for wound healing: a review of evidence from in vitro studies, animal experiments, and clinical trials. Int J Low Extrem Wounds 2005;4:23-44.

12. European Pressure Ulcer Advisory Panel, National Pressure Ulcer Advisory Panel. Treatment of pressure ulcers: quick reference guide. Washington: National Pressure Ulcer Advisory Panel, 2009.

13. Ricci E, Afaragan M. The effect of stochastic electrical noise on hard-to-heal wounds. J Wound Care 2010;19:96-103.

14. Jünger M, Arnold A, Zuder D, Stahl H, Heising S. Local therapy and treatment costs of chronic, venous leg ulcers with electrical stimulation (Dermapulse): a prospective, placebo controlled, double blind trial. Wound Repair Regen 2008;16:480-7.

15. Kitajo K, Nozaki D, Ward L, Yamamoto Y. Behavioral stochastic resonance within the human brain. Phys Rev Lett 2003;90: 218103.

16. Zeng F, Fu Q, Morse R. Human hearing enhanced by noise. Brain Res 2000;869(1-2):251-5.

17. Ricci E, Afargan M. Implication of stochastic resonance in a novel electrical frequency pattern associated with chronic wounds changes of pattern upon healing. EWMA $J 2010$.

18. Ohry A, Goren O. The effects of BST electrostimulation on healing of pressure ulcers: results of a multi-center, double blind, randomized, placebo controlled study. EWMA J 2007 oral abstract no. 66.

19. Liu W, Lipstiz L, Montero-Odasso M, Bean J, Kerrigan D, Collins J. Noise-enhanced vibrotactile sensitivity in older adults, patients with stroke, and patients with diabetic neuropathy. Arch Phys Med Rehabil 2002;83:171-6.

20. Khaodhiar L, Niemi J, Earnest R, Lima C, Harry J, Veves A. Enhancing sensation in diabetic neuropathic foot with mechanical noise. Diabetes Care 2003;26:3280-3.

21. Cloutier R, Horr S, Niemi J, D’Andrea S, Lima C, Harry J, Veves A. Prolonged mechanical noise restores tactile sense in diabetic neuropathic patients. Int J Low Extrem Wounds 2009;8:6-10.

22. Janković A, Binić I. Frequency rhythmic electrical modulation system in the treatment of chronic painful leg ulcers. Arch Dermatol Res 2008;300:377-83.

23. Bartsch T, Goadsby P. Central mechanisms of peripheral nerve stimulation in headache disorders. Prog Neurol Surg 2011;24: $16-26$. 